\title{
Mobilité électrophorétique et polarité négative acquises par Trypanosoma congolense rendu chimiorésistant au Moranyl (Suramine sodique)
}

\author{
por J.-P. BERSON
}

D'un point de vue purement général et sans tenir compte des résultats contradictoires obtenus, on peut dire que l'examen électrophorétique des trypanosomes, ou plus exactement l'éfude de leurs charges électriques, est déjà vieux de plus d'un demi siècle (TRAUBE 19121915, HOBER 1914, SZENT-GYORGYI 19201921). En ce qui concerne Trypanosoma congolense, il ne semble pas que jusqu'à maintenant on ait pu lui attribuer une charge définie. Quant à savoir quel signe prennent les trypanosomes chimiorésistants à certains médicaments, on peut dire que la question n'est pas résolve, exception faite de quelques travaux : BROOM, BROWN ef HOARE 1936, 1937, 1939.

L'auteur s'est donc proposé de soumettre à l'électrophorèse deux souches de Trypanosoma congolense, l'une normale et l'autre chimiorésistante au Moranyl et de rechercher ainsi une différence éventuelle dans les charges électriques des parasites.

\section{MATÉRIEL ET MÉTHODE}

Une souche de Trypanosoma congolense provenant de l'Institut Pasteur de Paris a été entretenue sur souris et rats blancs, elle est utilisée pour rechercher si un trypanosome non chimiorésistant possède une charge électrıque.

La souche chimiorésistante au Moranyl dérive de la précédente et a été obtenue par une technique originale dont on a fal mention dans un travail antérieur $\left(^{*}\right)$. Au moment de l'expé-

(*) Voir article précédent.

Rev. Elev. Méd. vét. Pays trop. 1963, 16, no 1.

Reçu pour publication : février 1963. rimentation, la chımiorésistance est de l'ordre de $500 \mathrm{mg} / \mathrm{kg}$ de souris, c'est-à-dire qu'elle est maximale.

L'appareillage spécialement conçu pour l'expérience, et utilisé ici diffère sensiblement des montages classiques, c'est pourquoi il en a été donné une description sommaire (schéma 1).

La «cellule » est constituée par une aiguille de verre creuse et à parois minces, son diamètre extérieur est de $1 \mathrm{~mm}$ environ. Chaque extrémité de l'aiguille est enfonçée dans un tube de verre coudé à $90^{\circ}$ et de plus grand diamètre, plein de gélose préalablement fondue avec un électrolyte dans le but de la rendre conductrice. Bien que la gélose se «tienne» suffisamment par elle-même pour, ne pas refluer dans' le bac où plonge l'extrémité distale du coude, il a paru souhaitable d'obstruer l'ouverture par un tampon de coton hydrophile qui jove alors le rôle d'une mèche. Chaque coude plonge dans un bac contenant une solution électrolytique du type du liquide de Ringer dilué.

Le courant électrique continu arrive aux bacs $A$ et $B$ par deux électrodes de charbon. La différence de potentiel mesurée aux deux extrémités de l'aiguilie est de 8 à 9 volts par centimètre, elle se trouve être de 80 volts lorsque l'aiguille mesure $10 \mathrm{~cm}$.

La «cellule» est remplıe de la manière suivante: on prélève, au moyen d'une seringue à insuline contenant déjà $0,5 \mathrm{~cm}$ de liquide de Hanks, 2 à 3 gouttes de sang de la queve d'une souris fortement parasitée, les proportions de ce mélange sont arbitraires mais il importe de ne pas avoir trop de parasites en suspension. On monte ensuite une fine aiguille sur la seringue 
et agissant sur le piston on remplit le canal sub-capillaire de l'aiguille en évitant les bulles d'air.

Le $\mathrm{pH}$ du milieu est de 7 à 7,2. L'aspect de la cellule après l'expérimentation a été reproduit sur le schéma 2.

La tecture microscopique s'effectue de la manière suivante, l'aiguille de verre pleine du milieu contenant les parasites soumis à l'électrophorèse, est fragmentée en tronçons de 1 à $2 \mathrm{~cm}$ de longueur. Chacun de ces tronçons retient par capillarité une gautte du milieu, c'est alors
Le résultat enregistré est presque superposable à celui signalé ci-dessus. Un fort dépôt de globules rouges se trouve près du pôle + , il ne semble pas que le traitement au Moranyl ait modifié de quelque manière que ce soit la polarité des hématies. Cependant aucun comptage n'a été pratiqué.

20 Mobilité des trypanosomes.

a) Tryponosomes non chimiorésistonts.

Les différents prélèvements pratiqués tout le

\section{SCHEMA I}

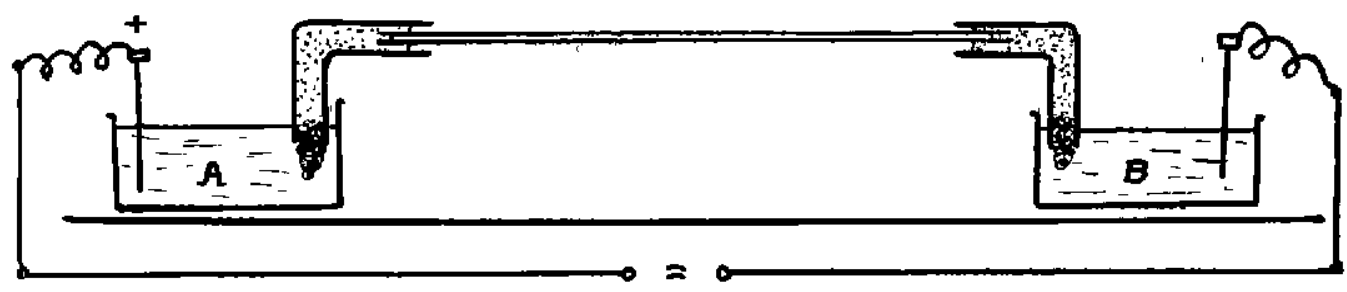

SCHEMA 2

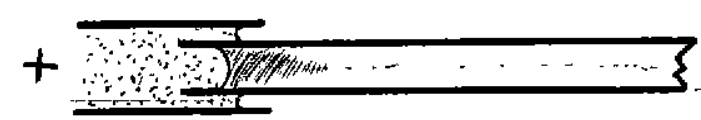

qu'à l'aide d'une pipette d̀ double effilure engagée dans le canal, on chasse la goutte sur une lame porte-objet.

\section{RÉSULTATS}

Jo Mobilité des hématies.

a) Sur souris parasitée par des trypanosomes non chimiorésistonts.

Le dépôt d'hématies trouvé au voisinage direct du pôle + est au moins deux fois plus important que celui trouvé près du pôle - - On rencontre également quelques hématies tout le long de la «cellule ».

b) Sur souris parositée par des tryponosomes chimiorésistants. long de la «cellule» ont montré une concentration des parasites à peu près constante et superposable à celle rencontrée avant le passage du courant, 5 à 6 par champ (Obj. $\times 40$ et Occ. $\times 8$ ).

b) Tryponosomes rendus chimiorésistants' au Moronyl.

90 p. 100 des trypanosomes ont été retrouvés au niveau du pôle positif, séparés de la gélose par le dépôt d'hématies, celles-ci semblant se déplacer plus rapidement que les parasites euxmêmes. Quelques trypanosomes ont été également mis en évidence au niveau du pôle négatif, enfin, dans le restant de la «cellule», quelques parasites vivants et morts ont pu être mis en évidence. 
Cette expérimentation a été répétée plusieurs fois, les résultats ont été similaires à ceux signalés ci-dessus. L'augmentation du temps de passage du courant ne semble pas modifier les résultats obtenus, la durée de marche a été en moyenne de 30 à 40 minutes. Mais il est possible qu'un temps d'électrophorèse plus court conduise aux mêmes constatations.

\section{DISCUSSION}

Avant d'entreprendre la discussion de ces résultats, il convient de préciser que WILLIAMSON (1960) a vaınement recherché un déplacement du point iso-électrique de deux fractions protéiques homogénéisées du cytoplasme de trypanosomes normaux et chimiorésistants. $\mathrm{Ce}$ qui laisse supposer que si la chimiorésistance détermine une modification électrique du parasite, cette dernière n'a pas lieu dans le cytoplasme. Or, le faił que l'on ait constaté un déplacement de l'ensemble d'un parasite chimiorésistant, conduit à penser que, si la charge d'uri trypanosome se trouve modifiée sans qu'on puisse mettre le cytoplasme en cause, c'est que cette modification a lieu au niveau même de la membrane. On peut donc dire qu'au moins en ce qui concerne le Moranyl, la chimiorésisłance entraîne une modification de la membrane du parasite, qui pourrait être à l'origine du changement de polarité du trypanosome.

Reste à savoir, bien entendu, pourquoi un trypanosome devenu chimiorésistant au Moranyl se trouve avoir une charge électrique modifiée pour ne pas dire spontanément acquise. Sans doute faut-il tenir compte du fait que le médicament est constitué de grosses molécules à 6 charges électriques négatives, et, de ce fait, le considérer comme un détergent anionique, négativant la membrane du parasite.

Les travaux de VON JANCSO (1952) et de ORMEROD et HILL (1956) (qui ont pu mettre en évidence des granulations de ce produit dans les cellules de l'organisme pour le premier, et dans les parasites eux-mêmes pour le second) permettent de supposer l'action interne du Moranyl, c'est-à-dire une action chimique et électrique sur le cytoplasme. On Ignore encore si l'action chimıque est réelle, quant à l'action élec- triq'ue, les travaux de WILLIAMSON semblent avoir mis le cytoplasme hors de cause, ce qui laisse supposer que la modification de charge esi supportée par la membrane.

D'après l'action électrique de surface telle que nous l'avons constatée, on peut penser que le parasite «vierge» $c^{\prime}$ est-à-dire non encore chimiorésistant, subit une imprégnation dès son premier contact avec le médicament; si la dose n'est pas suffisante pour le tuer il échappe au toxique, et, dans le même temps, sa membrane reçoit des charges négatives. Lors d'un deuxième traitement l'absorption du médicament par le parasite est freinée par la présence des premières charges déjà fixées et son accumulation dans le cytoplasme est moins aisée, permettant au trypanosome d'adapter son potentiel enzymatique au milieu dysgénésique ambiant; en même temps la membrane acquiert d'autres charges négatives. Au cours des traitements ultérieurs, les molécules de Moranyl ont d'autant plus de mal à pénétrer le parasıte que les charges négatives de sa membrane sont plus nombreuses, ce qui, pourrait-on dire, laısse le temps à la chimiorésistance «cytoplasmique» de' s'établir.

\section{CONCLUSION}

L'étude électrophorétique d'une souche de Trypanosoma congolense chimiorésistante du Moranyl a permis de reconnaître une polarité négative aux parasıtes. Cette polarité est sans doute liée à la membrane des trypanosomes. Il est évident que l'étude précise de la membrane des parasites permettrait de conclure plus sûrement.

Bien qu'à lui seul le phénomène électrique puisse expliquer l'acquisition de l'état de chimiorésistance, il est plus vraisemblable de considérer les deux faits comme séparés:

- Une modification électrique de la membrane, soit «désintéressée », soit directement liée à l'apparition de la chimiorésistance.

- Une modification du potentiel enzymatique, qui, comme chez les bactéries, adapte le métabolısme du parasite aux conditions dysgénésiques du milieu.

Institut d'Elevage et de Médecine vétérinoire des Pays tropicaux. 


\section{SUMMARY}

A study of the electrophoresis reaction of a Moranyl resistant strain of $T$. congolense

A comparative study with a normal strain of $T$. congolense and a strain chemoresistant to Suramin. The normal strain was neutral while the resistant strain was electro-negative. Contrary to the work of Williamson, this modification appeared to be due basıcally to the membrane of the parasites.

\section{RESUMEN}

Movilidad electroforética y polaridad negativa adquirida por Trypanosoma congolense, convertido en quimicorresistente por el Moranyl

El autor ha procedido al estudio electroforético de una estirpe de Trypanosoma congolense normal y de una estirpe quimicorresistente a la Suramina sôdica. La estirpe normal ha dado un resultado neutro y la estirpe quimicorresistente electronegativa.

Por oposición a los trabajos de Williamson, se supone que esta módificación eléctrica tiene como base la membrana de los parósitos.

\section{BIBLIOGRAPHIE}

BERSON (J. P.). - Contribution à l'étude de Trypanosoma congolense; sa biologie et sa chimiorésistance au Moranyl et à l'Antrycide pro-salt. Thèse de Doctorat Vétérinaire. Lyon, 1962.

$\operatorname{BROOM}(J . C),. \operatorname{BROWN}(H$. C.), HOARE (C. A.). - Studies in microcataphoresis. The electric charge of hemoflagellates. Trans. Roy. Soc. Trop. Med. Hyg. 193630 (1) : 87.

BROOM (J, C.), BROWN (H.C.), HOARE (C. A.). - Studies in trypanosomiasis. The electric charge of irypanosomes in Tsetse flies. Trans. Roy. Soc. Trop. Med. Hyg. 1937, 3 I (1).

BROOM (J. C.), BROWN (H. C.), HOARE (C. A.). - Studies in trypanosomiasis. The electric charge of trypanosomes in the salivary glands of Tsetse flies. Trans. Roy. Soc. Trop. Med. Hyg. 1939, 32 (4) : 545.

HOBER (R.). - - Beitrag zur physikalischen chemie der Vitalfärbung. Biochem. Z. 1914, $67: 420-30$.

JANCSO (N. ef A. von). - Suramin (Bayer 205) in animal tissues. Demonstration of Bayer 205 in tissues and its cellular distribur. Nature 1952, $170: 567$.

ORMEROD (W. E.). - - A study of basophilic inclusionbodies produced by chemotherapeutic agents in trypanosomes. Brit. J. Pharmocol. 1951, 6 : 334-41.
ORMEROD (W. E.). - Changes produced by trypanocid drugs in the phase contrast appearence of trypanosomes. Trans. Roy. Soc. Trop. Med. Hyg. 1956, 50 (4).

SZENT GYORGYI (A, von). - Eine mikroskopische uberführunggmethode. Blochem. $Z$. 1920, $110: 116-8$.

SZENT GYORGYI (A. von). - Kataphoreseversuche an Kleinlewesen. Studien über Eiweissreaktionen. Biochem. Z. 1921, II3: 29-35.

SZENT GYORGYI (A. von). - Beitrage zur physikalischen der Agglutination. Studien über Eiweissreaktionen. Biochem. Z. 1921, $113: 36-41$.

TRAUBE (J.). - Uber Arzneimittel und Gifte. Dische. Med. Wschr. 1912, $38: 1441-3$.

TRAUBE (J.). - Bemerkungen zu der Mitteilung von R. HOBER : Beitrag zur physikalischen chemie der Vitalfarbung. (Citation française de COMMANDON 1911). Blochem. Z. 1915, $69: 308-12$.

WILLIAMSON (J.). - Certains problèmes de la résistance à la médication trypanocide. (Traduction française du rapport de la ge réunion du Comité Scientifique International de Recherches sur les Trypanosomiases. VomJos. Nigeria du Nord). 1960. 